\title{
Experimental comparison of two multiple-stage contest designs with asymmetric players
}

\author{
Robin Chark • Amnon Rapoport • Rami Zwick
}

Received: 9 February 2009 / Accepted: 23 February 2010 / Published online: 7 March 2010

(C) The Author(s) 2010. This article is published with open access at Springerlink.com

\begin{abstract}
We compare experimentally two contest designs. In the between-group design (BGD), cohorts compete with one another in stage 1 and a single cohort is then advance to stage 2 . In stage 2 , members of this cohort compete for a single prize. In the withingroup design (WGD), the order of the competition is reversed. Our findings support the theoretical conclusion that the WGD is superior to BGD in generating more expenditure. They also show that if the cohorts are fixed, small, and interact repeatedly over time, then tacit collusion in the WGD, but not the BGD, leads to suppression of expenditures.
\end{abstract}

Keywords Two-stage contests $\cdot$ Asymmetric players $\cdot$ Laboratory experiments $\cdot$ Contest design

\section{Introduction}

Theoretical and experimental research on contests (see Konrad 2009, for a recent and comprehensive review) has focused on imperfectly discriminating contests ${ }^{1}$ with a single stage and symmetric agents. However, as noted by, among others, Amegashie (1999); Amegashie et al. (2007); Blavatskyy (2004), and Stein and Rapoport (2004, 2005), contests are often designed as multi- rather than single-stage competitions. In the preliminary stages contestants are grouped into independent cohorts where they compete with one another for the right to participate in the next stage. In the final stage, the winners of the previous stages compete with one another to secure one or more exogenously determined prizes. Representative

\footnotetext{
${ }^{1}$ In perfectly discriminating contests, also known as first-price all-pay auctions, the agent expending the greatest resource wins the competition and secures the highest prize. In imperfectly discriminating contests, the agent expending the greatest resource is assigned only the highest probability of winning the prize.
}

R. Chark

Hong Kong University of Science and Technology, Hong Kong, China

A. Rapoport · R. Zwick ( $\varangle)$

University of California, Riverside, USA

e-mail: ramiz@ucr.edu 
examples include various sport competitions, $R \& D$ contests, political races, and science contests.

Further examination of naturally occurring multi-stage contests shows that asymmetry of the contestants is the rule rather than exception. Asymmetry may be caused by heterogenous valuations of the prize, variability between agents in budget constraints, incomplete information about the cost of effort, different group sizes, or some combination of the above. In most sport competitions, science competitions, military conflicts, and political races, contestants differ from one another in their "ability." Based on their previous performance or initial resources, contestants are often categorized as favorites and underdogs or ranked in terms of their ex-ante chances of winning. This is expressed, for example, by the different odds placed by the media or gambling rings on the different contestants, by prior 'seeding' of NCAA teams and players in post-season tournaments, and by the surprise expressed by the media when the underdog wins the competition. When contestants differ in their ability, "upsets" are clearly unavoidable and may simply express a statistical illusion due to the probabilistic nature of the rule used to determine the winner. Harbaugh and Klumpp (2005) have studied whether this pattern might have a real foundation in the strategic allocation of resources by asymmetric agents. Che and Gale $(1997,1998,2003)$ have considered the effects of budget constraints on asymmetric players. Harstad (1995) examined a model with asymmetric informed agents in the context of winner-takes-all game. Wärneyrd (2003) studied a Bayesian model of rent-seeking contests with asymmetric information about the common prize. In Fey (2008), asymmetry between agents results from the incomplete information that they have about each other's cost of effort, and in Stein's (2002) model of single stage contests asymmetry is due to different group sizes and variability in valuations.

Theoretical analyses of contests have mostly been conducted with the purpose of contributing to contest design (see, e.g., Gradstein 1998; Gradstein and Konrad 1999; Moldovanu and Sela 2001, 2006). For example, Moldovanu and Sela (2001) studied the effects of the prize structure on the design of R\&D contests and concluded that in order to maximize the expected sum of expenditures, the designer should organize a winner-takeall contest if the contestants have linear or concave cost functions, but if they have convex cost functions two or more prizes may be optimal. Stein and Rapoport (2004) compared the properties of two contest designs; the Between- and Within-Group Designs. In the BetweenGroup Design (BGD), cohorts first compete with one another in stage 1 by expending resources. Depending on the expenditures in each cohort, a single cohort is chosen to advance to stage 2 . In stage 2 , members of this cohort compete with one another by expending additional resources and the same contest success function is implemented to award a single contestant an exogenously determined prize. Contests among regions (states) competing to secure the location of a new government-owned facility are often structured in such a way. Once a region is awarded the facility, each member of the region proceeds to compete with other members for the location of the facility. In the Within-Group Design (WGD), ${ }^{2}$ the order of the competition is reversed: in stage 1, cohorts act independently of one another with members of each cohort competing with one another by expending resources to receive a "ticket" for advancing to stage 2 . Then, the winners of stage 1 (one from each cohort) advance to stage 2 in which they compete with one another by expending additional resources. The same probabilistic contest success function is used to choose the ultimate winner who receives the prize. Beauty pageants, Rhodes scholars competitions, and post-season playoffs are often structured in such a way.

\footnotetext{
${ }^{2}$ Stein and Rapoport (2004) refer to this design as the "semi-finals" design. For ease of exposition we will refer to this design as the "Within-Group" design.
} 
Stein and Rapoport (2004) concluded that the WGD is superior to the BGD from the perspective of the contest designer if her goal is to generate the greatest expenditure by the contestants. Our position is that the implications of such recommendations for contest design depend in part on the actual behavior of flesh-and-blood contestants. If, in fact, the equilibrium solutions are upheld in experiments, then we have reason to believe that the theory yields practical implications that are valid. But if the equilibrium solution consistently fails to account for the observed patterns of behavior, then the significance of the design recommendations is compromised. There is a need for carefully conducted experiments in the controlled environment of the laboratory that implement the models' assumptions because abstract models are not designed for, nor can they fully capture, many of the features and nuances of naturally occurring contests.

Previous Experimental Research. Sharing the same perspective, there is a large body of experimental studies designed to test the implications of various models of contests. Included in this literature are the studies by Abbink et al. (2007), Amaldoss and Rapoport (2009), Amegashie et al. (2007), Anderson and Stafford (2003), Davis and Reilly (1998), Millner and Pratt (1989, 1991), Önçüler and Croson (2004), Parco et al. (2005), Potters et al. (1998), Schmitt et al. (2004), Schmitt et al. (2003), Shogren and Baik (1991), Vogt et al. (2002), and Weimann et al. (2000). Three major findings are shared by studies that examined the two-stage WGD contests. First, the experimental results deviate from equilibrium play. In particular, observed expenditures in stage 1 significantly exceed the equilibrium predictions. Perhaps there is an added utility gained for advancing to stage 2 in addition to and independently of the utility associated with winning the prize. Second, discrepancies between observed and predicted expenditures tend to diminish, though not entirely disappear, with iterations of the two-stage contest. Third, most experiments report considerable individual differences in expenditures that do not diminish with experience.

All of these experiments have studied symmetric players. In contrast, we focus on twostage contests with asymmetric players. Asymmetry is achieved by dividing the contestants into unequal group (cohort) sizes. As described below, we compare two contest designs that differ in the rules determining which players take part in stage 2 of the contest. A major and testable implication of the equilibrium solutions for these two designs is that not all the cohorts should necessarily expend resources in the first stage. Moreover, which cohorts take part in the contest and which do not depends on the contest design. This is the main hypothesis that we set out to test.

The rest of the paper is organized as follows. Sections 2 and 3 describe the two alternative contest designs, present the equilibrium solutions, and specify the numerical predictions for the parameter values incorporated in the experiment. Section 4 describes the experimental procedure and the results, and Section 5 concludes.

\section{The between-group design (BGD)}

There are $N$ risk-neutral players who compete with one another in a two-stage contest for a single exogenously determined and indivisible prize. The $N$ players initially are divided into $k$ cohorts with $n_{j}$ players in cohort $j\left(j=1, \ldots, k ; \sum_{j=1}^{k} n_{j}=N\right)$. In stage 1 of the contest, member $i$ of cohort $j$ expends $x_{i j}$ effort $\left(x_{i j} \geq 0\right)$. The sum of stage 1 expenditures over all $n_{j}$ members of cohort $j$ is denoted by $X_{j}=\sum_{i=1}^{n_{j}} x_{i j}$.

After resources are expended, a single cohort, say cohort $j$, is chosen to advance to stage 2 by comparing $X_{j}$ to the total expenditure across all the $k$ cohorts, $X=\sum_{j=1}^{k} X_{j}$. Thus, the ratio $X_{j} / X$ is the probability that cohort $j$ advances to stage 2 . In stage 2 , members 
of the winning cohort compete against one another by expending additional resources. In particular, each of its members expends an additional effort $y_{i j}\left(y_{i j} \geq 0\right)$. Denote the sum of stage 2 expenditures of all the $n_{j}$ members of the winning cohort by $Y_{j}$. Then, with probability $y_{i j} / Y_{j}$ member $i$ of the winning cohort $j$ is chosen to receive a prize valued at $V_{j}$.

\subsection{Equilibrium}

Without loss of generality, assume that the $k$ cohorts are ordered such that

$$
\frac{V_{1}}{n_{1}^{2}} \geq \frac{V_{2}}{n_{2}^{2}} \geq \cdots \geq \frac{V_{k}}{n_{k}^{2}}>0 .
$$

Because the $n_{j}$ members of cohort $j$ are symmetric, we simplify notation and denote the amount expended by each member of cohort $j$ by $x_{j}$. Then (Stein and Rapoport 2004), in equilibrium

$$
x_{j}=\frac{(p-1) H_{p}}{p n_{j}}\left[1-\frac{n_{j}^{2}(p-1) H_{p}}{p V_{j}}\right] \text {, }
$$

where

$$
H_{p}=\left[\frac{1}{p} \sum_{j \leq p} \frac{n_{j}^{2}}{V_{j}}\right]^{-1}
$$

is the harmonic mean of the $\left\{V_{j} / n_{j}^{2}\right\}$ from $j=1$ to $j=p$, and $p$ is an integer such that

$$
X_{1} \geq X_{2} \geq \cdots \geq X_{p}>0 \quad \text { and } \quad X_{j}=0 \quad \text { for } j>p .
$$

We say that $p$ is the number of active cohorts that expend non-negative amounts in stage 1 . $p$ is the largest integer index that satisfies

$$
\frac{V_{p}}{n_{p}^{2}}>\frac{(p-2) H_{p-1}}{p-1} .
$$

Equation (2) implies that $p \geq 2$. Thus, if the $N$ players are divided into $k=2$ cohorts, then both will participate in stage 1 . But if $k>2$, then one or more cohorts may be rendered inactive.

Assume that cohort $j$ is chosen by the probabilistic contest success function to advance to stage 2 . Then, in equilibrium, all the $n_{j}$ members of this cohort expend the same amount in stage 2 . Denote the amount expended by every player of cohort $j$ by $y_{j}$. Then,

$$
y_{j}=\frac{\left(n_{j}-1\right) V_{j}}{n_{j}^{2}} .
$$

This, in fact, is the same equilibrium solution as the one obtained for the standard singlestage rent-seeking model with common valuation $V$ and symmetric players. Each member of cohort $j$ wins the prize with probability $y_{j} / Y_{j}\left(=1 / n_{j}\right)$.

Finally, in equilibrium, the expected payoff for player $i$ of cohort $j$ at stage 1 , continuing optimally on to stage 2 , is given by

$$
E_{1}^{i j}(\underline{x}, \underline{y})=-x_{i j}+\frac{X_{j} V_{j}}{X n_{j}^{2}},
$$


Table 1 Equilibrium predictions for models BGD and WGD

\begin{tabular}{lllllc}
\hline Condition & Cohort & $x_{j}$ & $y_{j}$ & $P_{j}$ & $E_{1}^{i j}(\underline{x}, \underline{y})$ \\
\hline BGD & $n_{1}=3$ & 2.370 & 88.889 & 0.8 & 33.185 \\
& $n_{2}=6$ & 0.296 & 55.556 & 0.2 & 1.926 \\
& $n_{3}=9$ & 0 & 39.506 & 0 & 0 \\
\multirow{2}{*}{ WGD } & $n_{1}=3$ & 9.878 & 88.889 & $1 / 3$ & 4.937 \\
& $n_{2}=6$ & 6.173 & 88.889 & $1 / 6$ & 1.234 \\
& $n_{3}=9$ & 4.390 & 88.889 & $1 / 9$ & 0.548 \\
\hline
\end{tabular}

In both models the common valuation of the prize is $V=400$

$x_{j}$ Unconditional individual expenditures in stage 1

$y_{j}$ Expenditures in stage 2, conditional on optimal expenditures in stage 1

$P_{j}$ Probability of advancing to stage 2 (cohort in Condition BGD and individual player in Condition WGD) $E_{1}^{i j}(\underline{x}, \underline{y)}$ Expected payoff for player $i$ of cohort $j$ at stage 1, conditional on equilibrium behavior in stage 2

where $\underline{x}=\left(x_{1}, x_{2}, \ldots, x_{n_{j}}\right)$ and $\underline{y}=\left(y_{1}, y_{2}, \ldots, y_{n_{j}}\right)$.

It follows from (1) and (2) that the probability that cohort $j$ wins stage 1 is

$$
P_{j}=\frac{X_{j}}{X}=1-\frac{n_{j}^{2}}{V_{j}},
$$

and the probability that a specified member of cohort $j$ wins the prize is $W_{j}=P_{j} / n_{j}$.

\subsection{Predictions for the experiment}

In the experiment that we describe in Sect. $4, k=3, n_{1}=3, n_{2}=6$, and $n_{3}=9$. Although the equilibrium solution allows for different valuations of the prize, we have simplified the task by assuming a common valuation, namely, $V_{1}=V_{2}=V_{3} \equiv V=400$. Consequently, the only source of asymmetry is the difference in cohort size. Arranging the three cohorts by the values of the ratio $V / n_{j}^{2}$, we place them in the order cohort $1(n=3)$, cohort 2 $(n=6)$, and cohort $3(n=9)$. Computing $H_{j}$ for $j=1,2,3$, from (2), and then solving for $p$ from (3), we find that $p=2$. This implies that cohorts 1 and 2 participate in the contest but cohort 3 , which includes half of the players, does not. The individual expenditures for stage 1, computed from (1), are $x_{1}=2.370$ and $x_{2}=0.296$ for a total stage 1 expenditure of $X_{1}=7.112$ and $X_{2}=1.778$ for cohorts 1 and 2, respectively. This implies that cohort 1 advances to stage 2 with probability 0.8 and cohort 2 with the complementary probability 0.2 . Conditional on cohort $j(j=1,2)$ advancing to stage 2 , the individual expenditures computed from (4) are $y_{1}=88.889$ and $y_{2}=55.556$. The equilibrium expected payoffs computed from (5) are $E_{1}^{i 1}=33.185$ and $E_{1}^{i 2}=1.926$. Clearly, $E_{1}^{i 3}=0$. The top panel of Table 1 summarizes the equilibrium predictions.

\section{The within-group design (WGD)}

Similarly to model BGD, there are $N$ risk-neutral players who compete with one another for an exogenous prize. The $N$ players are divided into $k$ cohorts with $n_{j}$ players in cohort $j$. 
We denote the stage 1 expenditure of player $i$ in cohort $j$ by $x_{i j}$, and the sum of stage 1 expenditures of all members of cohort $j$ by $X_{j}$.

In contrast to BGD, in WGD a single player in each cohort is chosen to advance to stage 2 by comparing her expenditure to the sum of her cohort expenditures. Thus, player $i$ of cohort $j$ advances to stage 2 with probability $x_{i j} / X_{j}\left(=1 / n_{j}\right)$. In stage 2 , the $k$ winners of stage 1 compete by expending additional resources. Denote the expenditure of the player who advanced to stage 2 from cohort $j$ by $y_{j}$, and the sum of stage 2 expenditures by $Y=\sum_{j=1}^{k} y_{j}$. Then, with probability $y_{j} / Y(=1 / k)$, the player who advanced from cohort $j$ is chosen to receive the prize.

\subsection{Equilibrium}

The $k$ cohorts are ordered in terms of the magnitude of the prize valuations: $V_{1} \geq V_{2} \geq \cdots \geq$ $V_{k}>0$ (the numbering is arbitrary if all the prizes are equal). The ordering of the cohorts in WGD may not correspond to that in BGD. Only $m$ of the $k$ cohorts $(2 \leq m \leq k)$ participate in stage 1 and, subsequently, choose $m$ players to advance to stage 2 . To state the equilibrium solution (Stein and Rapoport 2004), it is convenient to start with stage 2.

In equilibrium, the number of active cohorts in stage 1 , denoted by $m$, is the largest integer that satisfies

$$
V_{m}>(m-2) \Phi_{m-1} /(m-1),
$$

where

$$
\Phi_{m}=\left[\frac{1}{m} \sum_{j \leq m} 1 / V_{j}\right]^{-1} .
$$

The equilibrium expenditure in stage 2 is given by

$$
y_{j}= \begin{cases}\frac{(m-1) \Phi_{m}}{m}\left[1-\frac{(m-1) \Phi_{m}}{m V_{j}}\right], & \text { if } j \leq m \\ 0, & \text { otherwise }\end{cases}
$$

where $\Phi_{m}$ and $m$ are computed from (7) and (6), respectively. This one-stage model was also solved by Hillman and Riley (1989) and Stein (2002).

Turning back to stage 1 , where individual expenditures within a cohort in stage 1 are equal, we can write $x_{j}$ instead of $x_{i j}$ to denote the individual expenditure of any member of cohort $j$ in stage 1 . We obtain

$$
x_{j}= \begin{cases}\frac{\left(n_{j}-1\right) V_{j} \pi_{j}^{2}}{n_{j}^{2}}, & \text { if } j \leq m \\ 0, & \text { otherwise }\end{cases}
$$

where $\pi_{j}=y_{j} / Y$. Thus, the equilibrium strategy is (9) in stage 1 for each member of cohort $j$ (for $j \leq m$ ) followed by (8) in stage 2 .

The expected payoff for player $i$ of cohort $j$ at stage 1 , continuing optimally on to stage 2 , is given by

$$
E_{1}^{i j}(\underline{x}, \underline{y})= \begin{cases}-x_{i j}+\frac{x_{i j} V_{j} \pi_{j}^{2}}{X_{j}}, & \text { if } j \leq m \\ 0, & \text { otherwise }\end{cases}
$$

and the probability that any member of cohort $j$ wins the prize is $W_{j}=\pi_{j} / n_{j}$. 


\subsection{Predictions for the experiment}

In our experiment, Conditions BGD and WGD share the same parameter values, namely, $k=3, n_{1}=3, n_{2}=6, n_{3}=9$, and a common prize valuation for all $N$ players of $V=400$. Model WGD uses the same contest success function as model BGD. Computing $\Phi_{m}$ for $j=1,2,3$, and subsequently solving for $m$ in (6), we obtain $m=3$. This means that, in contrast to model BGD, all three cohorts (total of 18 subjects) participate in stage 1 of the contest. The individual expenditures in stage 2, computed from (8), are the same for all $\mathrm{m}$ stage 1 winners, namely, $y_{1}=y_{2}=y_{3}=88.889$. The individual expenditures in stage 1 , computed from (9) for each cohort separately, are $x_{1}=9.878, x_{2}=6.173$, and $x_{3}=4.390$. Finally, the expected payoffs computed from $(10)$ are $E_{1}^{1 j}(\underline{x}, y)=4.937,1.234$, and 0.548 for $j=1,2$, and 3 , respectively (see bottom panel of Table 1 ).

\subsection{Comparing the two designs}

In evaluating the two models, it is instructive to compare them in terms of the expected rent dissipation over the two stages of the context. When the valuations of the prize are equal, as in the present study, the expected rent dissipation for BGD and WGD are computed from

$$
E(R D)=\frac{(p-1) V}{\sum_{j \leq p} n_{j}^{2}}\left[1-\sum_{j \leq p} n_{j}\left(n_{j}-1\right)\right]+V \sum_{j \leq p} \frac{n_{j}-1}{n_{j}}
$$

and

$$
R D=\frac{V(k-1)}{k}+\frac{V}{k^{2}} \sum_{j=1}^{k} \frac{n_{j}-1}{n_{j}},
$$

respectively. ${ }^{3}$ For the parameter values in the present experiment, we obtain $E(R D)=$ 288.889 and $R D=372.839$. When divided by the prize value $V=400$, the proportions of the prize expended under BGD and WGD are 0.722 and 0.932 , respectively. This is the basis for the conclusion reached by Stein and Rapoport (2004) that WGD is superior to BGD from the perspective of the contest designer in generating higher expenditures.

\section{Experiment}

\subsection{Method}

Subjects Two hundred and sixteen subjects participated in 12 separate experimental sessions, six sessions in Condition BGD and six in Condition WGD. Both male and female students responded in nearly equal numbers. There were 18 subjects in each session, both undergraduate and graduate students. The subjects were recruited by a computerized system that asked for volunteers to participate in a group decision making experiment for payoffs contingent on performance. Each session lasted about 90 minutes. The mean payoff per subject $^{4}$ was \$208 in Condition BGD and \$211 in Condition WGD.

\footnotetext{
${ }^{3}$ Note that whereas in WGD the number of contestants advancing to stage 2 is fixed at $k$, the number of contestants advancing to stage 2 in BGD is only probabilistically given hence only expected rent dissipation can be calculated for BGD.

${ }^{4}$ All amounts are in HK\$ (US\$1 $=$ HK\$7.8).
} 
Procedure All sessions were conducted at a large computerized laboratory. After being seated, the subjects received written instructions and proceeded to read them at their own pace. The Appendix presents the instructions for Condition BGD; the ones for Condition WGD are similar, except of the obvious changes, and are therefore omitted.

The subjects participated in 40 identically structured trials (rounds) that implemented the two-stage contest. At the beginning of the session, they were randomly assigned to three cohorts $1(n=3), 2(n=6)$, and $3(n=9)$. (In the instructions, these are called groups A, $\mathrm{B}, \mathrm{C}$, rather than cohorts $1,2,3$.). Cohort membership was held constant across all trials. To ensure that subjects did not lose money in the experiment and to prevent wealth effects, each subject started each round with a fixed endowment of $\$ 100$. In equilibrium, the $\$ 100$ endowment does not impose a binding budget constraint since the maximum total expenditure by any player in any of the cohorts and conditions does not exceed 98.767 (this is the expected total expenditure by members of cohort 1 in Condition WGD). Next, each subject decided privately and independently how much resources to expend in stage 1 , how much to expend in stage 2 (if reached), and how much money not to expend at all (provided the sum could not exceed \$100). Once all the subjects submitted their expenditure decisions, the computer selected the cohort (Condition BGD) that advanced to stage 2 or the $k=3$ stage 1 winners (Condition WGD), one from each group, who advance to stage 2. Next, in stage 2, the computer selected the winner (Champion). The rule used by the computer for selecting the stage 1 winners and for selecting the Champion has already been described in Sections 2 (BGD) and 3 (WGD). Resources expended by each subject in stages 1 and 2 of the contest were forfeited. At the end of the trial, each subject kept whatever was left of the $\$ 100$ that was not expended. The Champion also kept her award of $\$ 400$.

Subjects were provided full outcome information at the end of each round. In Condition BGD, after all subjects submitted their expenditures, the computer presented the individual expenditures of all subjects in all cohorts and the total allocation of the three cohorts (in stage 1) together with each cohort corresponding probabilities of winning stage 1 . After the winning cohort was announced, the computer displayed the probabilities of winning the contest for each individual member of the winning cohort. Next, the Champion was announced. In Condition WGD, after all subjects submitted their expenditures, the computer presented the individual expenditures of all subjects in all cohorts together with the probability of each player winning stage 1 within his/her own cohort. After the identity of the winner in each cohort was announced, the computer presented the probabilities of winning the contest for each player who advanced to the second stage. Next, the Champion was announced. The final Outcome screen informed each subject of her payoff in this trial. Once all subjects reviewed it, they independently pressed the "continue" button, and the experiment proceeded to the next round. A history screen was available at all times for subjects to review their own decisions and outcomes in all previous trials.

At the end of the session, two of the 40 trials were randomly selected for payment. As mentioned earlier, this payoff scheme was chosen to eliminate wealth effects, as the subjects only learned their payoffs at the end of the session. It resulted in considerable individual differences in the final payments. A subject expending her entire endowment on the two trials that were randomly chosen for payment would have ended up with a final payoff of $\$ 0$ if she did not win the prize on either trial. Otherwise, if she expended a small fraction of her endowment but happened to win the prize on one of these two payoff trials, she would have earned $\$ 500$ (minus her expenditures). If she happened to win on both payoff rounds, her final payoff would have been $\$ 1000$ (minus her expenditures). 


\subsection{Results}

\subsubsection{Mean expenditures in stage 1}

Given previous findings in multiple experiments on one- and two-stage contests that do not support the quantitative implications of the equilibrium solution (see Sect. 1), we mostly focus on its qualitative implications. To do so, we formulate and then test several hypotheses about mean expenditures between and within conditions. We denote the mean expenditure of a member of cohort $j(j=1,2,3)$ in stage 1 of Condition BGD by $x_{j B}^{(1)}$, and the mean expenditure of a member of cohort $j$ in stage 2 by $x_{j B}^{(2)}$. We use similar notation for denoting the corresponding mean expenditures in Condition WGD after substituting the subscript " $W$ " for " $B$ ". The equilibrium solutions for Conditions BGD and WGD give rise to the following three hypotheses about stage 1 expenditures:

$$
\begin{gathered}
\mathrm{H} 1: \quad x_{1 B}^{(1)}>x_{2 B}^{(1)}>x_{3 B}^{(1)} \\
\mathrm{H} 2: \quad x_{1 W}^{(1)}>x_{2 W}^{(1)}>x_{3 W}^{(1)} \\
\mathrm{H} 3: \quad x_{1 W}^{(1)}>x_{1 B}^{(1)}, \quad x_{2 W}^{(1)}>x_{2 B}^{(1)}, \quad x_{3 W}^{(1)}>x_{3 B}^{(1)} .
\end{gathered}
$$

Recall that the subjects were assigned to the same cohort in all 40 rounds of play. This design feature was introduced to facilitate convergence of individual expenditures with experience in playing the game. We divided the 40 rounds into four blocks of 10 rounds each and tested hypotheses $\mathrm{H} 1$ through $\mathrm{H} 3$ in the fourth block. In later sections we report results of the dynamics of play across all 40 rounds; however, for testing hypotheses implied by a static solution concept, subjects' more stable decisions after obtaining considerable experience with the game are preferred.

Table 2 presents the means and standard deviations of the individual expenditures by condition (BGD and WGD), cohort (1,2, and 3), and stage (1 and 2) in the last block of trials. We subjected the mean expenditures of each cohort in the last block to an ANOVA. The analysis was conducted separately for each combination of condition and stage. Cohort effect was significant in both conditions and both stages. The results of multiple comparisons between cohorts within condition and stage are presented in Table 2 . To the right of each mean there is a bracket containing one or two digits indicating the cohort(s) that are significantly different from each other. ${ }^{5}$

The multiple-comparison test provides evidence in support of $\mathrm{H} 1$. The mean expenditure of cohort 1 in Condition BGD is significantly higher than the mean expenditures of cohort 2, which, in turn, is significantly higher than the mean expenditure of cohort 3 .

The equilibrium solution implies that all of the nine members of cohort 3 in Condition BGD should stay inactive. This prediction is not self-evident and might require reasoning that is not expected from subjects with bounded rationality. Subjects in cohort $3\left(n_{3}=9\right)$ might have reasoned that given their largest group size they should collectively expend more resources than the other smaller groups and thereby increase the probability of their cohort advancing to stage 2 . This reasoning might have motivated members of this cohort to expend positive resources in stage 1 rather than completely withdraw from the contest as predicted. Forward-looking reasoning might have anticipated the within-cohort competition in stage 2 and consequently the low probability of winning the contest. Together with the opportunity

\footnotetext{
${ }^{5}$ An effect is marked as significant if $p \leq 0.016$ for a family-wise (session) type I error of 0.05 .
} 
Table 2 Mean observed expenditure in block 4 by condition, cohort, and stage

\begin{tabular}{|c|c|c|c|c|c|c|c|}
\hline & & Stage 1 & & & Stage 2 & & \\
\hline & & Cohort & & & Cohort & & \\
\hline & & $1(n=3)$ & $2(n=6)$ & $3(n=9)$ & $1(n=3)$ & $2(n=6)$ & $3(n=9)$ \\
\hline BGD & Mean & $7.98[2,3]$ & $3.25[1,3]$ & $1.8[1,2]$ & $36.16[2,3]$ & $51.76[1,3]$ & $29.18[1,2]$ \\
\hline & Std & 16.97 & 7.92 & 5.2 & 43.26 & 39.68 & 42.68 \\
\hline WGD & Mean & $3.62[2,3]$ & $5.61[1,3]$ & $8.14[1,2]$ & $67.19[2,3]$ & $50.89[1]$ & $54.46[1]$ \\
\hline & Std & 8.55 & 8.76 & 10.97 & 40.71 & 44.4 & 41.73 \\
\hline
\end{tabular}

of free riding in stage 1 it might have dampened this motivation. As reported in Table 2, members of cohort 3 expended, on average, less than members of cohorts 1 and 2, but the mean expenditure (1.8) is by no means zero as predicted. However, and in agreement with the equilibrium prediction, additional analysis that focuses on individual expenditures shows that $78.3 \%$ of the members of cohort 3 completely withdrew from the contest (expended zero) in stage 1 in the last block.

The equilibrium solution also implies that members of cohort 2 in Condition BGD expend slightly less that $0.3 \%$ of their endowment in stage 1 . This implication seems as implausible as the prediction of zero expenditure for members of cohort 3 . However, it was also supported by the data. Two-thirds of the subjects in cohort $2(66.2 \%)$ expended zero in the last block. In other words, the large majority of the members of cohorts 2 and 3 (15 of 18 subjects) in Condition BGD expended $\$ 0$ or less than $\$ 1$ in stage 1, providing strong evidence in support of equilibrium play.

The multiple-comparison analysis refutes H2. In fact, the opposite trend is observed than predicted. The mean expenditure of cohort 1 in Condition WGD (3.62) is significantly lower than the mean expenditure of cohort 2 (5.62), which, in turn, is significantly lower than the mean expenditure of cohort 3 (8.14). The explanation that we propose invokes the notion of tacit coordination which is not accounted for by the equilibrium solution. Contestants try to win stage 1 within a cohort by expending minimum effort. Given that winning a stage is a function of the relative rather than absolute expenditure, each contestant in stage 1 of Condition WGD is motivated to expend more than others. At the same time, cohort members may benefit by tacitly agreeing to suppress their expenditures on this stage (thereby leaving more money to compete in stage 2). ${ }^{6}$ Because it is easier to achieve tacit coordination in smaller than larger cohorts if the two-stage game is repeated, this hypothesis leads to the directional results of stage 1 expenditures in Condition WGD. Evidence in support of this behavioral explanation is provided later when we examine the dynamics of play.

We turn next to hypothesis H3, which compares expenditures between rather than within conditions. Mean expenditures presented in Table 2 indicate that $\mathrm{H} 3$ is supported in two out of the three cohorts. Contrary to the equilibrium prediction, mean expenditures of cohort 1 in stage 1 of Condition BGD (7.98) is significantly higher than the corresponding mean in Condition WGD (3.62). However, H3 is supported in both cohorts 2 and 3 that together account for $83.3 \%$ of all subjects (3.25 vs. 5.61 for cohort 2 in Conditions BGD and WGD, respectively; and 1.8 vs. 8.14 for cohort 3 in Conditions BGD and WGD, respectively). The

\footnotetext{
${ }^{6}$ Such motivation can also exist in condition BGD but the coordination needs to be achieved among the three cohorts and not just among the players of a single cohort. Such cross-cohort coordination can hardly be expected.
} 
latter two pairs of means are significantly different from each other $(p<0.05)$. The failure of $\mathrm{H} 3$ correctly to predict the relative expenditures of cohort 1 in Conditions BGD and WGD is also attributed to the fact that the three players of cohort 1, after gaining substantial experience with the task, could tacitly agree to expend smaller amounts in Condition WGD but not in Condition BGD where such behavior is not beneficial.

Focusing on the quantitative values of stage 1 expenditures (in block 4), we find that in contrast to previously published results (that implement WGD only), on average, not all cohorts in Condition WGD exhibited excess expenditures. In particular, cohort $1(n=3)$ expended on average significantly less than predicated (3.62 vs. 9.88, respectively, $p<0.01$ ), cohort $2(n=6)$ expended at about the predicted level (5.61 vs. 6.17, respectively, $p>0.1)$. Only cohort $3(n=9)$ expended on average more than predicted ( 8.14 vs. 4.39 , respectively, $p<0.05)$. In the discussion section we provide an explanation for this deviation from previous studies. The results of Condition BGD are consistent with previous studies. All the three cohorts expended on average more than predicted (compare Tables 1 and 2-all differences are significant at the 0.05 level).

\subsubsection{Mean expenditures in stage 2}

The contestants were asked to submit their stage 2 expenditures conditional on advancing to stage 2 before receiving outcome information about stage 1 expenditures of their cohort members. Therefore, in both conditions, stage 2 decisions could not be affected by the outcomes of stage 1 (in the same round). This design feature allows the analysis of stage 2 expenditures of all 18 subjects in the session, not only of the subjects who actually advanced to stage 2 . Using the same notation as above, with the superscript " 2 " for stage 2 replacing the subscript " 1 " for stage 1 , the equilibrium solution (Table 1) implies the following three hypotheses:

$$
\begin{array}{ll}
\mathrm{H} 4: & x_{1 B}^{(2)}>x_{2 B}^{(2)}>x_{3 B}^{(2)} \\
\mathrm{H} 5: & x_{1 W}^{(2)}=x_{2 W}^{(2)}=x_{3 W}^{(2)}
\end{array}
$$

$$
\text { H6: } \quad x_{1 W}^{(2)}=x_{1 B}^{(2)}, \quad x_{2 W}^{(2)}>x_{2 B}^{(2)}, \quad x_{3 W}^{(2)}>x_{3 B}^{(2)} .
$$

The multiple-comparison analysis provides evidence in partial support of $\mathrm{H} 4$. Table 2 shows that, as implied by the equilibrium solution, the hypothesis that $x_{2 B}^{(2)}>x_{3 B}^{(2)}$ is supported (significantly), but contrary to the prediction mean expenditure in stage 2 is lower in cohort 1 than cohort $2\left(x_{1 B}^{(2)}<x_{2 B}^{(2)}\right)$. We account for this deviation from equilibrium prediction by invoking the same reasoning that we attributed to the subjects to account for the finding that members of cohort 1 in Condition WGD could tacitly agree to lower their expenditures in stage 1 . Note that the three players of cohort 1 in Condition BGD, if they advance to stage 2 , find themselves in exactly the same situation as the three players of cohort 1 in stage 1 of Condition WGD. That is, each player is motivated to expend more than others, but at the same time all cohort members benefit from tacitly agreeing to suppress their expenditures. The fixed-matching nature of the design and the small group size $(n=3)$ facilitated the ability of cohort 1 members to coordinate their expenditures when such coordination was beneficial.

The multiple-comparison analysis also provides evidence in partial support of H5. Table 2 shows that, as implied by the equilibrium solution, the hypothesis that $x_{2 W}^{(2)}=x_{3 W}^{(2)}$ cannot be rejected. However, contrary to the prediction, the mean expenditures in stage 2 is higher for cohort 1 than cohorts $2\left(x_{1 W}^{(2)}>x_{2 W}^{(2)}\right)$ and $3\left(x_{1 W}^{(2)}>x_{3 W}^{(2)}\right)$. Recall that we have 
reported that cohort 1's mean expenditure in stage 1 in Condition WGD was lower than in the other two cohorts, in contradiction to the equilibrium analysis. We have proposed a behavioral explanation of these results in terms of the ability of a small cohort tacitly to lower the expenditures of its members. Given the informational structure of the game, members of this cohort were aware that the stage 1 winner from this group advanced to stage 2 by expending, on average, less than stage 1 winners from the other two groups. Consequently, stage 1 winners from cohort 1 might have believed that with more "left over" resources, they were in a better position to win stage 2 if, for example, every finalist expends whatever is left after advancing to stage 2 . Indeed, the percentage of games where cohort 1 members allocated the entire endowment of $\$ 100$ to stages 1 and 2 (combined) in the last 10 rounds is $65.5 \%$, whereas the corresponding percentages for cohorts 2 and 3 are only 44.7 and 48.5 , respectively.

The multiple-comparison analysis shows that only one of the predicted relationships in H6 holds (e.g., $x_{3 W}^{(2)}>x_{3 B}^{(2)}$ ). Table 2 shows that, in contrast to the equilibrium analysis, the

hypothesis $x_{1 W}^{(2)}=x_{1 B}^{(2)}$ is rejected in favor of the alternative hypothesis $x_{1 W}^{(2)}>x_{1 B}^{(2)}$. This difference sheds light on the players' ability to tacitly coordinate their expenditures. Note that in both Conditions BGD and WGD a member of cohort 1 , if advancing to stage 2 , finds herself in a similar contest with two other players; hence, the equilibrium solution of equal expenditures (of cohort 1) in stage 2 in both conditions. However, from a behavioral point of view (and the current fixed-matching design), the identity of the other two players is critical. Whereas in Condition BGD the other two players are always members of the same cohort, in Condition WGD they come from different cohorts. Consequently, if they advance to stage 2 in Condition BGD, then the three players of cohort 1 can tacitly agree to lower their expenditures, whereas the three players in stage 2 of Condition WGD cannot. This may account for the finding $x_{1 W}^{(2)}>x_{1 B}^{(2)} .^{7}$

\subsubsection{Comparing overall rent dissipation}

Stein and Rapoport (2004) concluded that model WGD is superior to model BGD in generating more expenditure. This conclusion is largely based on the expected expenditures in stage 1. Contestants on this stage in our study are expected to expend a total of 8.886 and 106.182 in Conditions BGD and WGD, respectively (see Table 1). In stage 2, the total expected expenditure is slightly higher in Condition BGD (280) than Condition WGD (266.7) mainly because there is a $20 \%$ chance in the former condition that six (cohort 2) rather than three (cohort 1) players will advance to stage 2. Nevertheless, the total sum of expenditures over stages is expected to be higher in Condition WGD (372.8) than Condition BGD (288.9).

Hypothesis $\mathrm{H} 3$ compared the mean individual expenditure in stage 1 by condition within cohort. We have already reported that cohorts 2 and 3 expended significantly more resources in Condition WGD than Condition BGD, but that cohort 1 exhibited the opposite pattern. Nevertheless, given that cohort 1 is the smallest, the total sum of expenditures over the three cohorts in stage 1 is, as predicted, significantly higher in Condition WGD than Condition BGD (117.78 vs. 59.64, respectively, $p<0.05$ ).

Hypothesis H6 compared the mean individual expenditure in stage 2 by condition within cohort. We have reported that consistent with equilibrium play, cohort 3 expended significantly more resources in Condition WGD than Condition BGD. Contrary to the prediction,

\footnotetext{
${ }^{7}$ Of course, all players can observe the behavior of all other players, no matter if they are from their own cohort or not. Nevertheless, we argue that actual experiences and the ability to reciprocate other players' behavior in the next trial is the main facilitator of tacit coordination and not just "observational learning".
} 
the same pattern holds for cohort 1, and cohort 2 did not expend more resources in Condition WGD than Condition BGD. Taken together, these results might have indicated that contestants expended more in stage 2 of Condition WGD than Condition BGD; however, the means here are somewhat misleading. Recall that exactly three contestants advance to stage 2 in Condition WGD, one from each cohort, and expend additional resources. However, any cohort in Condition BGD may advance to stage 2; hence, the number of stage 2 contestants who expend resources on this stage varies from three (if cohort 1 advances) to nine (if cohort 3 advances) thereby affecting the total expenditure. The equilibrium predicts (for Condition BGD) that the probability that each cohort will advance to stage 2 is $0.8,0.2$, and 0.0 , for cohorts 1,2 , and 3, respectively. However, the corresponding observed probabilities (in the last block) were $0.433,0.317$, and 0.250. In other words, cohort 3 advanced to stage 2 more often then predicted and cohort 1 less often. Calculating the total expenditure in stage 2 under both conditions, we found that consistent with this prediction, the total expenditure is higher in Condition BGD (211.1) than Condition WGD (172.5). This difference is only marginally significant $(p<0.08)$. Summing over both stages, our results support the conclusion of Stein and Rapoport (2004) that contestants expend more resources in Condition WGD than Condition BGD. In fact, the total expenditures in the last block were 290.3 and 270.7, for Conditions WGD and BGD, respectively. The difference between these sums is marginally significant $(p<0.06)$. Note that the total expenditure in Condition BGD is close to the predicted value of 288.9. However, the total expenditure in Condition WGD is lower than predicted (372).

4.2.3.1 Dynamics of play in stage 1 The equilibrium solution is a static concept that cannot account for the effects of learning from experience that we find in the present study. For all cohorts and all sessions, we find that with experience subjects decrease their expenditures in stage 1. In addition, we find persistent sequential dependencies in the expenditures. To better understand the dynamics of play, we have examined the effects of the following two variables on the decision to expend equal, fewer, or additional resources on the next round:

$$
\begin{aligned}
& \operatorname{sign}\left(x_{i j t}-\overline{X_{j t}}\right): \begin{cases}>\bar{X}, & x_{i j t}>\overline{X_{j t}} \\
<\bar{X}, & x_{i j t}<\overline{X_{j t}} \\
=\bar{X}, & x_{i j t}=\overline{X_{j t}}\end{cases} \\
& \text { outcome of round } t:\left\{\begin{array}{l}
\text { L1-loses stage } 1 \\
W 1 \text { L2 -wins stage 1, loses stage 2 } \\
W 1 \text { W2-wins stage 1, wins stage 2 }
\end{array}\right.
\end{aligned}
$$

Table 3A presents the proportions of the decisions to expend equal, fewer, or more resources in stage 1 of the next round as a function of the above two variables. The first column of the table shows whether the subject expended (in stage 1) more $(>\bar{X})$, less $(<\bar{X})$ or the same resources $(=\bar{X})$ as the cohort's average on round $t$. The second column indicates whether the subject expended (in stage 1) less $(\downarrow)$, more $(\uparrow)$, or the same $(=)$ resources on round $t+1$ compared to round $t$. The columns labeled L1, W1L2, and W1W2 present the three possible outcomes of round $t$. The numbers in bold indicate the number of observations in each case. For example, consider cohort 3 in Condition BGD: in 460 cases $(264+161+35)$ members of this cohort expended more resources than the cohort's average on round $t$. In 264 cases, the subjects did not advance to stage 2 (L1), in 161 cases they advanced to stage 2 but did not win the contest (W1L2), and in 35 cases they won the contest. Out of the 161 cases in which the subjects advanced to stage 2 but did not win the contest, in $66 \%$ of the 
Table 3A Proportions of decisions to expend equal, fewer, or more resources on the next round of play. Stage 1

\begin{tabular}{|c|c|c|c|c|c|c|c|c|c|c|}
\hline \multirow[t]{3}{*}{$x_{j i t}$} & \multirow[t]{3}{*}{$x_{i j t+1}$} & \multicolumn{9}{|c|}{ Cohort } \\
\hline & & \multicolumn{3}{|c|}{$1(n=3)$} & \multicolumn{3}{|c|}{$2(n=6)$} & \multicolumn{3}{|c|}{$3(n=9)$} \\
\hline & & L1 & W1L2 & W1W2 & L1 & W1L2 & W1W2 & $\overline{\mathrm{L} 1}$ & W1L2 & W1W2 \\
\hline \multicolumn{11}{|l|}{ BGD } \\
\hline \multirow[t]{4}{*}{$>\bar{X}$} & & 155 & 42 & 50 & 278 & 109 & 32 & 264 & 161 & 35 \\
\hline & $\downarrow$ & 0.35 & 0.64 & 0.42 & 0.43 & 0.62 & 0.44 & 0.50 & 0.66 & 0.40 \\
\hline & $\uparrow$ & 0.30 & 0.07 & 0.26 & 0.19 & 0.07 & 0.16 & 0.19 & 0.03 & 0.20 \\
\hline & $=$ & 0.35 & 0.29 & 0.32 & 0.37 & 0.30 & 0.41 & 0.31 & 0.30 & 0.40 \\
\hline \multirow[t]{4}{*}{$<\bar{X}$} & & 237 & 107 & 26 & 643 & 234 & 38 & 947 & 533 & 52 \\
\hline & $\downarrow$ & 0.06 & 0.07 & 0.23 & 0.03 & 0.08 & 0.05 & 0.02 & 0.04 & 0.04 \\
\hline & $\uparrow$ & 0.20 & 0.15 & 0.19 & 0.17 & 0.10 & 0.13 & 0.10 & 0.09 & 0.17 \\
\hline & $=$ & 0.74 & 0.78 & 0.58 & 0.80 & 0.82 & 0.82 & 0.88 & 0.87 & 0.79 \\
\hline \multirow[t]{4}{*}{$=\bar{X}$} & & 79 & 5 & 1 & 63 & 7 & $\mathbf{0}$ & 112 & 2 & $\mathbf{0}$ \\
\hline & $\downarrow$ & 0.04 & 0.20 & 0.00 & 0.06 & 0.43 & & 0.02 & 0.00 & \\
\hline & $\uparrow$ & 0.29 & 0.20 & 1.00 & 0.21 & 0.00 & & 0.22 & 0.50 & \\
\hline & $=$ & 0.67 & 0.60 & 0.00 & 0.73 & 0.57 & & 0.76 & 0.50 & \\
\hline \multicolumn{11}{|c|}{ WGD } \\
\hline \multirow[t]{4}{*}{$>\bar{X}$} & & 148 & 107 & 51 & 403 & 134 & 67 & 726 & 115 & 75 \\
\hline & $\downarrow$ & 0.61 & 0.65 & 0.53 & 0.41 & 0.44 & 0.40 & 0.44 & 0.55 & 0.45 \\
\hline & $\uparrow$ & 0.09 & 0.05 & 0.02 & 0.22 & 0.10 & 0.12 & 0.20 & 0.14 & 0.19 \\
\hline & $=$ & 0.30 & 0.31 & 0.45 & 0.37 & 0.46 & 0.48 & 0.36 & 0.31 & 0.36 \\
\hline \multirow[t]{4}{*}{$<\bar{X}$} & & 295 & 48 & 13 & 760 & 21 & 9 & 1137 & 28 & 16 \\
\hline & $\downarrow$ & 0.13 & 0.15 & 0.15 & 0.08 & 0.38 & 0.22 & 0.09 & 0.29 & 0.31 \\
\hline & $\uparrow$ & 0.15 & 0.13 & 0.15 & 0.26 & 0.05 & 0.22 & 0.24 & 0.14 & 0.13 \\
\hline & $=$ & 0.73 & 0.73 & 0.69 & 0.66 & 0.57 & 0.56 & 0.67 & 0.57 & 0.56 \\
\hline \multirow[t]{4}{*}{$=\bar{X}$} & & 25 & 13 & 2 & 7 & 2 & 1 & 9 & 0 & 0 \\
\hline & $\downarrow$ & 0.40 & 0.38 & 0.00 & 0.29 & 0.00 & 0.00 & 0.33 & & \\
\hline & $\uparrow$ & 0.24 & 0.23 & 0.00 & 0.29 & 0.00 & 0.00 & 0.22 & & \\
\hline & $=$ & 0.36 & 0.38 & 1.00 & 0.43 & 1.00 & 1.00 & 0.44 & & \\
\hline
\end{tabular}

time the subjects invested fewer resources on round $t+1$ (compared to round $t$ ), and in only $3 \%$ of these cases did they expend more (and in $30 \%$ of the cases they expended the same amount).

Several relationships can be observed in Table 3A and will be verified below as statistically significant.

(1) In both conditions and all cohorts, independently of the outcome of round $t$, the subjects who expended more than the cohort's average on round $t$ were more likely to expend less on round $t+1$ (compare $\downarrow$ and $\uparrow$ under " $>\bar{X}$ ").

(2) In condition BGD and in all cohorts, the subjects who expended less than the cohort's average on round $t$ were more likely to expend more on round $t+1$, independently of the outcome of round $t$ (the only exception is cohort 1 in Condition BGD under the W1L2 case). In Condition WGD, because subjects who expended less than the cohort's 
average were less likely to advance to stage 2, most of the observations are for those subjects who did not advance to stage 2 (L1). In these cases, subjects were more likely to expend more on round $t+1$ (compare $\uparrow$ and $\downarrow$ under " $<\bar{X}$ ").

(3) In both conditions and in all cohorts, independently of the outcome of round $t$, the subjects were much more likely to expend the same amount on round $t+1$ as on round $t$, if on round $t$ they expended less than the cohort's average (compare " $=$ " under " $<\bar{X}$ " vs. under " $>\bar{X}$ ").

These three behavioral regularities jointly account for the general trend of decreasing expenditures in stage 1 as the contest stage game is iterated in time.

Observation (1) above describes a main effect. However, the tendency to expend less on round $t+1$ after expending more than the cohort's average on round $t$ interacts with the results of round $t$. That is, observation (1) is much more pronounced if the cohort advanced to stage 2 but the subject did not win the contest (W1L2) compared to when the cohort did not advance to stage 2 (L1). Subjects who expended more than the cohort's average contributed disproportionally to the cohort's probability of winning stage 1 and advancing to stage 2 . These subjects probably believed that the other members of their cohort were free riding on their own contribution, and these beliefs intensified if the cohort indeed advanced to stage 2 but another cohort member (who might have expended less in stage 1) won the contest (W1L2). A similar interaction is not observed in condition WGD. Clearly, there is no opportunity in this condition to free ride.

We reported previously that, on average, cohort 1 in Condition WGD expended less than cohorts 2 and 3 in stage 1 in the same condition. We conjectured that the small cohort size facilitated coordination in lowering the expenditures by members of this cohort. Table $3 \mathrm{~A}$ provides evidence in support of this conjecture. The trend toward suppression of expenditures, as reported above, is similar in all cohorts; however, it is much more pronounced in cohort 1 of Condition WGD. After expending more than the cohort's average, members of cohort 1 are much more likely to expend less on round $t+1$ and are less likely to expend more in comparison to members of cohorts 2 and 3 in the same condition. Independently of the outcome of round $t$, the percentages for cohort 1 are $61 \%$ (less) and $6.4 \%$ (more). The corresponding percentages for cohort 2 are $41.5 \%$ (less) and $18.2 \%$ (more), and for cohort 3 they are $45.5 \%$ (less) and $19.2 \%$ (more). Similarly, after expending less than the cohort's average, members of cohort 1 are more likely to expend less on round $t+1$, and are less likely to expend more, compared to members of cohorts 2 and 3 in the same condition. ${ }^{8}$ Independently of the outcome of round $t$, the percentages for cohort 1 are $13.3 \%$ (less) and $14.7 \%$ (more). The corresponding percentages for cohort 2 are $8.9 \%$ (less) and $25.4 \%$ (more), and for cohort 3 they are $9.8 \%$ (less) and $23.6 \%$ (more). Taken together, these results show that the tendency to decrease the expenditure after expending more than the average is stronger in cohort 1 than in the other two cohorts, and that the tendency to increase the expenditure after expending less than the cohort average is weaker in cohort 1 than in the other two cohorts. This accounts for the relatively low expenditure level of cohort 1 in Condition WGD.

4.2.3.2 Dynamics of play in stage 2 Table 3B presents the proportions of the decisions in stage 2 to expend equal, fewer, or more resources on the next round of stage 2 as a function of (1) the outcome on round $t$ and (2) the relationship between one's own expenditure in stage 2 on round $t$ and a reference group average. In Condition BGD, the relevant reference group is one's cohort. In condition WGD, the relevant reference group is the mean expenditure (in stage 2) of the three players (one from each cohort) who advanced to stage 2 on round $t$.

\footnotetext{
${ }^{8}$ This statement refers to case L1 that includes most of the observations.
} 
Table 3B Proportions of decisions to expend equal, fewer, or more resources on the next round of play. Stage 2

\begin{tabular}{|c|c|c|c|c|c|c|c|c|c|c|}
\hline \multirow[t]{3}{*}{$x_{j i t}$} & \multirow[t]{3}{*}{$x_{i j t+1}$} & \multicolumn{9}{|c|}{ Cohort } \\
\hline & & \multicolumn{3}{|c|}{$1(n=3)$} & \multicolumn{3}{|c|}{$2(n=6)$} & \multicolumn{3}{|c|}{$3(n=9)$} \\
\hline & & L1 & W1L2 & W1W2 & L1 & W1L2 & W1W2 & L1 & W1L2 & $\mathrm{W} 1 \mathrm{~W} 2$ \\
\hline \multicolumn{11}{|l|}{ BGD } \\
\hline \multirow[t]{4}{*}{$>\bar{X}$} & & 246 & 42 & 71 & 494 & 148 & 58 & 552 & 266 & 73 \\
\hline & $\downarrow$ & 0.62 & 0.43 & 0.45 & 0.32 & 0.34 & 0.22 & 0.31 & 0.42 & 0.37 \\
\hline & $\uparrow$ & 0.18 & 0.36 & 0.30 & 0.18 & 0.31 & 0.22 & 0.23 & 0.26 & 0.15 \\
\hline & $=$ & 0.20 & 0.21 & 0.25 & 0.50 & 0.35 & 0.55 & 0.46 & 0.32 & 0.48 \\
\hline \multirow[t]{4}{*}{$<\bar{X}$} & & 208 & 108 & 5 & 489 & 200 & 12 & 766 & 429 & 14 \\
\hline & $\downarrow$ & 0.14 & 0.16 & 0.20 & 0.16 & 0.15 & 0.33 & 0.07 & 0.11 & 0.29 \\
\hline & $\uparrow$ & 0.21 & 0.22 & 0.40 & 0.33 & 0.42 & 0.08 & 0.17 & 0.27 & 0.07 \\
\hline & $=$ & 0.65 & 0.62 & 0.40 & 0.52 & 0.44 & 0.58 & 0.76 & 0.62 & 0.64 \\
\hline \multirow[t]{4}{*}{$=\bar{X}$} & & 17 & 4 & 1 & 1 & 2 & 0 & 5 & 1 & 0 \\
\hline & $\downarrow$ & 0.35 & 0.25 & 0.00 & 1.00 & 0.00 & & 0.20 & 0.00 & \\
\hline & $\uparrow$ & 0.24 & 0.25 & 1.00 & 0.00 & 1.00 & & 0.40 & 1.00 & \\
\hline & $=$ & 0.41 & 0.50 & 0.00 & 0.00 & 0.00 & & 0.40 & 0.00 & \\
\hline \multicolumn{11}{|c|}{ WGD } \\
\hline \multirow[t]{4}{*}{$>\bar{X}$} & & 336 & 123 & 66 & 675 & 141 & 76 & 1154 & 128 & 85 \\
\hline & $\downarrow$ & 0.24 & 0.25 & 0.17 & 0.23 & 0.28 & 0.24 & 0.26 & 0.30 & 0.27 \\
\hline & $\uparrow$ & 0.31 & 0.42 & 0.24 & 0.36 & 0.35 & 0.25 & 0.33 & 0.41 & 0.29 \\
\hline & $=$ & 0.45 & 0.33 & 0.59 & 0.41 & 0.37 & 0.51 & 0.41 & 0.29 & 0.44 \\
\hline \multirow[t]{4}{*}{$<\bar{X}$} & & 129 & 45 & 0 & 493 & 14 & 0 & 716 & 13 & 6 \\
\hline & $\downarrow$ & 0.13 & 0.24 & & 0.07 & 0.06 & & 0.04 & 0.07 & 0.00 \\
\hline & $\uparrow$ & 0.34 & 0.29 & & 0.26 & 0.50 & & 0.25 & 0.55 & 0.50 \\
\hline & $=$ & 0.53 & 0.47 & & 0.67 & 0.44 & & 0.71 & 0.38 & 0.50 \\
\hline \multirow[t]{4}{*}{$=\bar{X}$} & & 3 & 0 & 0 & 2 & 2 & 1 & 2 & 2 & 0 \\
\hline & $\downarrow$ & 0.00 & & 0.00 & 0.00 & 0.00 & 0.00 & 0.00 & & \\
\hline & $\uparrow$ & 0.33 & & 0.00 & 0.00 & 0.00 & 0.50 & 1.00 & & \\
\hline & $=$ & 0.67 & & 1.00 & 1.00 & 1.00 & 0.50 & 0.00 & & \\
\hline
\end{tabular}

The same dynamics that were observed in stage 1 are observed in stage 2 and will be verified below as statistically significant.

(1) In both conditions and in all cohorts, independently of the outcome of round $t$, subjects who expended more than the relevant reference group on round $t$ were much more likely to expend less on round $t+1$ (compare $\downarrow$ and $\uparrow$ under " $>\bar{X}$ ").

(2) In both conditions and in all cohorts, independently of the outcome of round $t,{ }^{9}$ subjects who expended less than the relevant reference group on round $t$ were much more likely to expend more on round $t+1$ (compare $\uparrow$ and $\downarrow$ under " $<\bar{X}$ ").

\footnotetext{
${ }^{9} \mathrm{~W} 1 \mathrm{~W} 2$ outcome is excluded from this statement because only a very few observations fall in this category.
} 
Table 4 ANOVA results ( $p$-values)

\begin{tabular}{|c|c|c|c|c|c|c|}
\hline & \multicolumn{3}{|l|}{ Stage 1} & \multicolumn{3}{|l|}{ Stage 2} \\
\hline & \multicolumn{3}{|l|}{ Cohort } & \multicolumn{3}{|l|}{ Cohort } \\
\hline & $1(n=3)$ & $2(n=6)$ & $3(n=9)$ & $1(n=3)$ & $2(n=6)$ & $3(n=9)$ \\
\hline \multicolumn{7}{|l|}{ BGD } \\
\hline $\operatorname{sign}\left(x_{i j t}-\bar{X}\right)$ & 0.047 & $<.0001$ & $<.0001$ & $<.0001$ & $<.0001$ & $<.0001$ \\
\hline Outcome $(t)$ & 0.138 & 0.063 & 0.302 & 0.901 & 0.313 & 0.155 \\
\hline Interaction & 0.018 & 0.132 & 0.005 & 0.958 & 0.699 & $<.0001$ \\
\hline \multicolumn{7}{|l|}{ WGD } \\
\hline $\operatorname{sign}\left(x_{i j t}-\bar{X}\right)$ & $<.0001$ & $<.0001$ & $<.0001$ & $<.0001$ & $<.0001$ & $<.0001$ \\
\hline Outcome $(t)$ & 0.057 & 0.034 & 0.206 & 0.650 & 0.159 & 0.886 \\
\hline Interaction & 0.967 & 0.022 & 0.332 & 0.922 & 0.076 & 0.940 \\
\hline
\end{tabular}

(3) In both conditions and in all cohorts, independently of the outcome of round $t$, subjects were much more likely to expend the same amount on round $t+1$ as on round $t$ if they expended less than the reference group on round $t$ compared to when they expended more than the reference group (compare " =" under " $<\bar{X}$ " vs. under " $>\bar{X}$ ").

4.2.3.3 Statistical analysis Table 4 presents the $p$-values of a repeated measure ANOVA with $\left(x_{i j t+1}-x_{i j t}\right)$ as the dependent variable and $\operatorname{sign}\left(x_{i j t}-\bar{X}\right),{ }^{10}$ and outcome of round $t(\mathrm{~L} 1, \mathrm{~W} 1 \mathrm{~L} 2)^{11}$ as the independent variables. Observations (1) and (2) above are statistically significant for all cohorts and for both stages (see the $p$-values of the $\operatorname{sign}\left(x_{i j t}-\bar{X}\right)$ variable). Observation (3) above for Condition BGD in stage 1 is significant in cohorts 1 and 3 and marginally significant in cohort 2 .

\section{Discussion}

There is an inherent difficulty in testing the equilibrium solution in games that are not perceived as trivial. On the one hand, it is by now commonly accepted that the equilibrium solution is not achieved by intuition. Rather, if reached at all, it is by some process of learning. Hence, the design feature of iterating the game multiple times until behavior stabilizes. On the other hand, learning introduces sequential dependencies that are not accounted for by the static solution concept. Cognizant of this difficulty, our approach has been to use the equilibrium solution as a benchmark and test its predictive power on the last block of trials when it is more likely that the expenditures have stabilized. We have complemented this test with a behavioral explanation proposed to account for the major deviations from equilibrium on the aggregate level. Perhaps more importantly, we have supported this explanation by the analysis of the dynamics of play across all the 40 rounds.

Our results show that differences in contest design matter. Consistent with Stein and Rapoport's (2004) theoretical predictions, observed overall expenditures in Condition WGD are higher than in Condition BGM. If, indeed, the contest designer's goal is to elicit higher

\footnotetext{
${ }^{10}$ The categories of " $<\bar{X}$ " and " $=\bar{X}$ " were combined to eliminate empty cells in the analysis.

${ }^{11}$ The categories W1L2 and W1W2 were combined to eliminate empty cells in the analysis.
} 
expenditures, our experimental results corroborate the theoretical predictions that Condition WGD is better suited for this purpose. However, our findings that members of cohort 1 in Condition WGD could tacitly coordinate their low expenditures in stage 1 point to a potential abuse of this design in small cohorts.

The analysis of mean expenditures in the last block provides only partial support for the qualitative predictions, more so in stage 1 than in stage 2 . When deviations from equilibrium are observed, they may be accounted for by simple psychological reasoning that is not part of the equilibrium analysis. Focusing on asymmetry between players due to different cohort size, most members of the two largest cohorts in Condition BGD learned to stay out of the contest in stage 1. In Condition WGD, where a single member from each cohort advances to stage 2, expenditure levels in stage 1 were considerably higher than in Condition BGD. To account for the conflict between the desire to advance to stage 2 and to minimize stage 1 expenditures, we proposed an explanation in terms of tacit agreement to lower stage 1 expenditures. The hypothesis that cohort size is negatively correlated with the success of reaching such agreement seems natural. The analysis of sequential dependencies, that played no role in formulating this hypothesis, strongly supports it.

We find no evidence in Condition WGD for over-expenditure in stage 1 for cohorts 1 and 2. Only cohort 3 exhibited over-expenditure. We attribute this finding to the difference between the fixed-matching design that we used in the present study and the variablematching design that was used in previous studies (i.e., Amaldoss and Rapoport 2009, and Parco et al. 2005). The fixed-matching design facilitates the contestants' ability to tacitly coordinate low expenditures in stage 1. However, this is possible mainly in small groups; hence, the results that members of cohort 3 expended more than predicted is consistent with this interpretation. Recall that in both Amaldoss and Rapoport, and Parco et al. each cohort included only two players. However, given the variable-matching design, cohort members could not easily coordinate suppression of expenditures in stage 1 .

Both matching design features have their merit. Our results should pertain to environments where cohorts are formed for the long run and their members participate repeatedly in contests for external resources. Examples include different departments within a university that compete for external grants. The contest design could either be BGD or WGD, but membership in a department is typically fixed for a long term and departments participate in multiple contests over time. Another example would be annual competition between school districts for extra state funding. As school districts are fixed, fixed- rather than variablematching designs would prove to be more appropriate.

Ours is one of the first experimental studies of contests with asymmetric players. More studies are called for which manipulate cohort size, number of cohorts, valuations of the prize, and magnitude of the budget constraint. Not only may they shed light on behavior in contests, where asymmetry is the rule rather than exception, but they may also help in evaluating conclusions regarding contest design that at present are based solely on theory.

Acknowledgements We gratefully acknowledge financial support from the Hong Kong Research Grant Commission (RGC grant HKUST6307/04H).

Open Access This article is distributed under the terms of the Creative Commons Attribution Noncommercial License which permits any noncommercial use, distribution, and reproduction in any medium, provided the original author(s) and source are credited.

\section{Appendix: Subject instructions}

This experiment has been designed to study how people compete in a two-stage tournament. The winner of the tournament wins $\mathbf{\$ 4 0 0}$. The rules of the tournament are explained below. 


\section{A.1 General description of the tournament}

You are one of $\mathbf{1 8}$ players who are asked to compete in a two-stage tournament. At the beginning of the tournament, players will be placed into three groups named Groups A, B, and C. Group A contains 3 players, Group B contains 6 players, and Group C contains 9 players.

In the first stage, the three groups compete with one another by expending funds to determine the group that wins stage 1 . This group proceeds to stage 2 . Next, in stage 2 of the tournament, members of the winning group, which we call the finalists, will compete with one another by expending additional funds. The winner of stage 2 , whom we call the Champion, will be awarded a prize of $\$ 400$.

Here are the rules of the tournament. Each player starts the tournament with a fixed endowment of $\mathbf{\$ 1 0 0}$. Each player then decides privately and independently how much money to expend in stage 1, how much to expend in stage 2 (if reached), and how much money not to expend at all. The total amount expended in stages 1 and 2 cannot exceed $\$ 100$.

Once all the players have made their expenditure decisions, the computer will select the group that advances to stage 2 and the Champion from that group. The rule used by the computer for selecting the group that wins stage 1 and for selecting the Champion from this group will be explained below.

Money expended in stage 1 of the tournament is lost for all 18 players. Only the finalists (members of stage 1 winning group) may expend money in stage 2, and for them this money is lost. At the end of the tournament, each of the 18 players will keep whatever left of the $\$ 100$ that was not expended. The Champion, of course, will also keep his/her award of $\$ 400$.

\section{A.2 How stage 1's winning group is selected}

The computer will randomly choose the winning group based on probabilities of win that are determined by the decisions of the players in stage 1 .

Each group's probability of winning stage 1 is determined by the sum of the expenditures of the members of this group in comparison to the sum of the expenditures of all the players across the three groups. As long as the group's sum of expenditures is a positive amount in stage 1, that group has a chance of winning this stage. Specifically, the probability of a group winning stage 1 is calculated using the following formula:

$$
\text { A group's chances of winning }=\frac{\text { Amount expended by this group }}{\text { Total amount expended by ALL the } 3 \text { groups }} .
$$

\section{A.2.1 Example}

Suppose that each group expends the following amount in stage 1:

\begin{tabular}{ll}
\hline Group & Total stage 1 expenditure (\$) \\
\hline A & 10 \\
B & 42 \\
C & 30 \\
\hline
\end{tabular}

The total expenditure across the three groups in stage 1 is $\$ 82$. The probability that, say, Group B wins stage 1 is given by

$$
\text { Group B's probability of winning }=\frac{42}{10+42+30}=0.512 \text {. }
$$


Thus, Group B has a $51.2 \%$ chance of winning stage 1 . The probabilities of winning stage 1 by the other two groups are calculated in a similar way and are summarized in the table below:

\begin{tabular}{lll}
\hline Group & Total stage 1 expenditure (\$) & Chances of winning \\
\hline A & 10 & $12.2 \%$ \\
B & 42 & $51.2 \%$ \\
C & 30 & $36.6 \%$ \\
\hline
\end{tabular}

The computer will randomly choose the winning group based on the above probabilities.

\section{A.3 How the champion is selected}

The computer will randomly select the Champion based on the probabilities of win that are determined by the stage 2 expenditure decisions of the finalists.

Each finalist's chances of winning stage 2 and becoming the Champion are determined by his/her expenditure in comparison to the sum of the expenditures of all the other finalists in stage 2 . As long as a player expends a positive amount in stage 2 , he/she has some chance of winning this stage and becoming the champion. Specifically, the probability of winning stage 2 is calculated using the following formula:

A player's chances of winning stage 2

Amount expended by this player on Stage 2

$=\frac{\text { Total amount expended by ALL the players in her group on Stage } 2}{\text {. }}$

\section{A.3.1 Example}

Suppose that Group B won stage 1 and the 6 members of Group B decided to expend the following amounts in stage 2:

\begin{tabular}{ll}
\hline Player & Stage 2 expenditure $(\$)$ \\
\hline 1 & 18 \\
2 & 43 \\
3 & 3 \\
4 & 12 \\
5 & 8 \\
6 & 6 \\
\hline
\end{tabular}

The total expenditure of Group B in stage 2 is $\$ 90$. The probability that, say, Player 4 wins stage 2 and becomes the Champion is given by

$$
\text { Player 4's probability of winning }=\frac{12}{18+43+3+12+8+6}=0.133 \text {. }
$$

Thus, Player 4 has $13.3 \%$ chance of becoming the Champion. The probabilities of winning stage 2 by the other players in the group are calculated in a similar way and are presented in 
the table below:

\begin{tabular}{lcc}
\hline Player & Stage 2 expenditure (\$) & Chances of winning \\
\hline 1 & 18 & $20.0 \%$ \\
2 & 43 & $47.8 \%$ \\
3 & 3 & $3.3 \%$ \\
4 & 12 & $13.3 \%$ \\
5 & 8 & $8.9 \%$ \\
6 & 6 & $6.7 \%$ \\
\hline
\end{tabular}

The computer will randomly select the winner (Champion) from this group based on the above probabilities.

\section{A.4 Payoffs from the game}

If you are the Champion, your payoff will be $\$ 400$ plus whatever left of the $\$ 100$ that you didn't expend in stages 1 and 2.

If you are member of the group that won stage 1 but not the Champion, your payoff will be whatever left of the $\$ 100$ that you didn't expend in stages 1 and 2 .

If you are not a member of the winning group, then your payoff will be whatever left of the $\$ 100$ that you didn't expend in stage 1 .

\section{A.4.1 Description of the computer displays}

This is the screen that you will see at the beginning of each game (Fig. 1). Your task is to enter the amount you want to expend in stage 1, and the amount you want to expend in stage 2, if you reach this stage. You will enter these two amounts by typing the numbers in the corresponding boxes. After you enter both amounts and press ENTER, the computer will calculate the amount you decide to keep, namely, the amount you don't want to expend in the tournament. You can enter both amounts as many times as you wish. When you are satisfied with your decision, press the Submit button to submit your decision.

After all the players submit their decisions, the computer will display the results of stage 1. The display will look like the one on Fig. 2.

You will see how much money each player decided to expend in stage 1 , how much to expend in stage 2 (if reached), and how much money not to expend at all (kept). In addition, you will see the total expenditure of each group in stage 1 and the corresponding probabilities that each group will win stage 1 .

Next, the computer will select the winning group and the identity of the group will be displayed with the corresponding probabilities of wining the tournament for each member of this group. The display will look like the one on Fig. 3.

Next, the identity of Stage 2 winner will be displayed as well as the payoffs to all players. The display will look like the one on Fig. 4.

This concludes the game.

Before the next game starts, a screen showing your individual history of play up to this point will be displayed. 


\section{Game 1}

You are Player 1 in Group C

Group A has 3 players, B 6 players, and C 9 players

Each player starts the game with $\$ 100$

How much do you want to expend in each stage?

\begin{tabular}{|c|c|}
\hline Stage 1 & 1 \\
\cline { 2 - 2 } Stage 2 (if reached) & \\
\hline Keep & \\
\hline Total & $\$ 100$ \\
\hline
\end{tabular}

Fig. 1

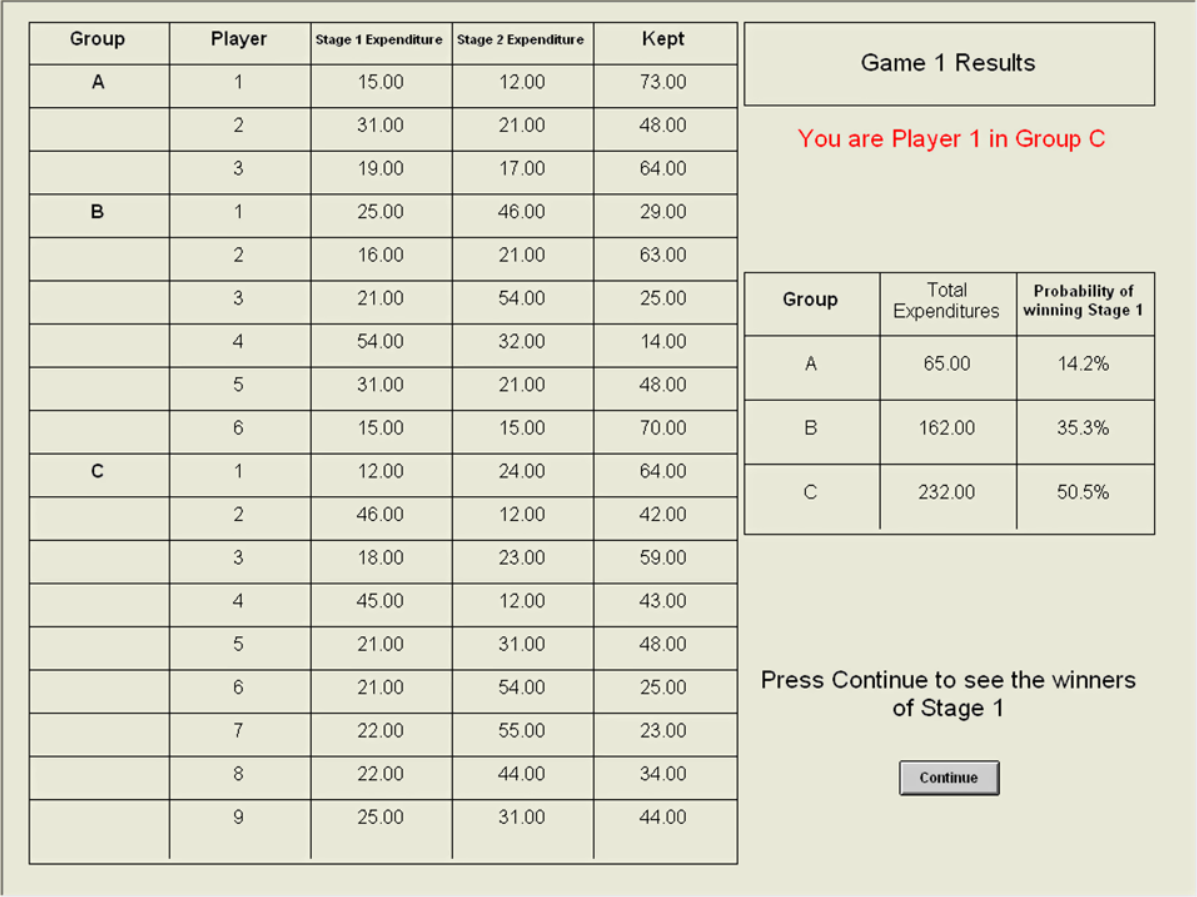

Fig. 2 


\begin{tabular}{|c|c|c|c|c|c|c|c|}
\hline Group & Player & Stage 1 Expenditure & Stage 2 Expenditure & Kept & \multirow{2}{*}{\multicolumn{3}{|c|}{ Game 1 Results }} \\
\hline \multirow[t]{3}{*}{ A } & 1 & 15.00 & 12.00 & 73.00 & & & \\
\hline & 2 & 31.00 & 21.00 & 48.00 & \multirow{2}{*}{\multicolumn{3}{|c|}{ You are Player 1 in Group C }} \\
\hline & 3 & 19.00 & 17.00 & 64.00 & & & \\
\hline \multirow[t]{6}{*}{ B } & 1 & 25.00 & 46.00 & 29.00 & \multirow{2}{*}{\multicolumn{3}{|c|}{ Group A wins Stage 1}} \\
\hline & 2 & 16.00 & 21.00 & 63.00 & & & \\
\hline & 3 & 21.00 & 54.00 & 25.00 & Player & \multicolumn{2}{|c|}{$\begin{array}{c}\text { Probability of winning } \\
\text { Stage } 2\end{array}$} \\
\hline & 4 & 54.00 & 32.00 & 14.00 & \multirow{2}{*}{1} & \multirow{2}{*}{\multicolumn{2}{|c|}{$24.0 \%$}} \\
\hline & 5 & 31.00 & 21.00 & 48.00 & & & \\
\hline & 6 & 15.00 & 15.00 & 70.00 & 2 & & $42.0 \%$ \\
\hline \multirow[t]{9}{*}{ c } & 1 & 12.00 & 24.00 & 64.00 & \multirow{2}{*}{3} & \multirow{2}{*}{\multicolumn{2}{|c|}{$34.0 \%$}} \\
\hline & 2 & 46.00 & 12.00 & 42.00 & & & \\
\hline & 3 & 18.00 & 23.00 & 59.00 & \multirow{7}{*}{\multicolumn{3}{|c|}{$\begin{array}{c}\text { Press Continue to see the winner of } \\
\text { Stage } 2\end{array}$}} \\
\hline & 4 & 45.00 & 12.00 & 43.00 & & & \\
\hline & 5 & 21.00 & 31.00 & 48.00 & & & \\
\hline & 6 & 21.00 & 54.00 & 25.00 & & & \\
\hline & 7 & 22.00 & 55.00 & 23.00 & & & \\
\hline & 8 & 22.00 & 44.00 & 34.00 & & & \\
\hline & 9 & 25.00 & 31.00 & 44.00 & & & \\
\hline
\end{tabular}

Fig. 3

\begin{tabular}{|c|c|c|c|c|c|}
\hline Group & Player & $\begin{array}{c}\text { Stage 1 } \\
\text { Expenditure }\end{array}$ & $\begin{array}{c}\text { Stage } 2 \\
\text { Expenditure }\end{array}$ & Kept & Payoffs \\
\hline \multirow[t]{3}{*}{ A } & 1 & 15.00 & 12.00 & 73.00 & 73.00 \\
\hline & 2 & 31.00 & 21.00 & 48.00 & 48.00 \\
\hline & 3 & 19.00 & 17.00 & 64.00 & 464.00 \\
\hline \multirow[t]{6}{*}{ B } & 1 & 25.00 & 46.00 & 29.00 & 75.00 \\
\hline & 2 & 16.00 & 21.00 & 63.00 & 84.00 \\
\hline & 3 & 21.00 & 54.00 & 25.00 & 79.00 \\
\hline & 4 & 54.00 & 32.00 & 14.00 & 46.00 \\
\hline & 5 & 31.00 & 21.00 & 48.00 & 69.00 \\
\hline & 6 & 15.00 & 15.00 & 70.00 & 85.00 \\
\hline \multirow[t]{9}{*}{ C } & 1 & 12.00 & 24.00 & 64.00 & 88.00 \\
\hline & 2 & 46.00 & 12.00 & 42.00 & 54.00 \\
\hline & 3 & 18.00 & 23.00 & 59.00 & 82.00 \\
\hline & 4 & 45.00 & 12.00 & 43.00 & 55.00 \\
\hline & 5 & 21.00 & 31.00 & 48.00 & 79.00 \\
\hline & 6 & 21.00 & 54.00 & 25.00 & 79.00 \\
\hline & 7 & 22.00 & 55.00 & 23.00 & 78.00 \\
\hline & 8 & 22.00 & 44.00 & 34.00 & 78.00 \\
\hline & 9 & 25.00 & 31.00 & 44.00 & 75.00 \\
\hline
\end{tabular}

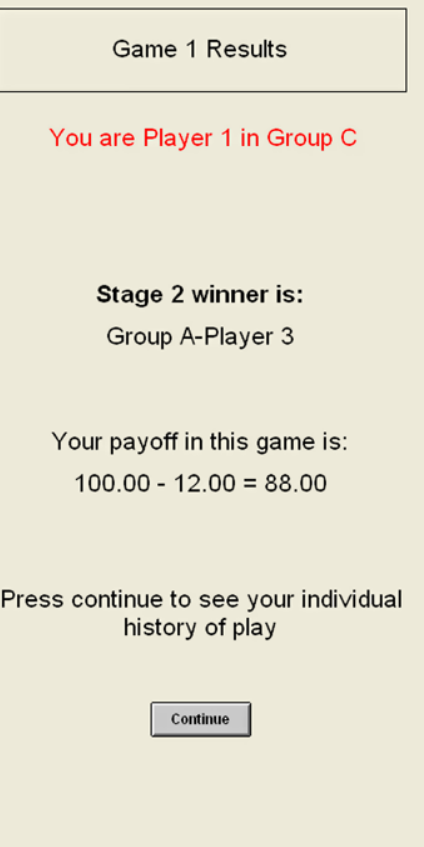

Fig. 4 


\section{A.4.2 Summary}

There are 18 players in this experiment who will play the same tournament game $\mathbf{4 0}$ times. Before the first game starts, the computer will divide the 18 players into three groups of 3 , 6 , and 9 players each. You will be a member of the same group for all 40 games.

Each game is a two-stage tournament. The winner of the tournament will receive $\$ 400$. In stage 1, the three groups will compete with one another to determine a single group that advances to stage 2 . Next, in stage 2, members of the winning group will compete with one another to determine the Champion. The Champion will receive a prize of $\$ 400$. Each player starts the game with an endowment of $\$ 100$. You have to decide how to allocate the $\$ 100$ between stage 1 and stage 2 (if reached). All the players will keep the amount they do not expend on the tournament. In addition, the Champion will be awarded a prize of $\$ 400$.

Note that each player faces the same problem. Because the budget is limited to $\$ 100$, the more you expend in stage 1, the higher the probability of your group advancing to stage 2 . However, the more you expend in stage 1, the less money you have to expend in stage 2 and the less money you can keep if your group fails to win stage 1 . Similarly in stage 2 , the more you expend on this stage, the higher is your probability of winning the $\$ 400$ prize. However, the more you expend in stage 2 the less money you keep if you fail to win this stage. Your problem is to balance these wins and losses while taking into consideration the expenditure decisions of all the other players.

\section{A.5 Payment at the end of the session}

At the end of the session, 2 games will be randomly selected out of the 40 . We will pay you in cash your cumulative payoffs from these games.

If you have any questions, please raise your hand and the monitor will assist you. Otherwise, please click on the "START" button on the screen to indicate that you have completed reading the instructions and are ready to start the game. Please wait patiently until all other players are ready to start.

\section{References}

Abbink, K., Brandts, J., Herrmann, B., \& Orzen, H. (2007). Inter-group conflict and intra-group punishment in an experimental contest game. CREED, University of Amsterdam, Unpublished manuscript.

Amaldoss, W., \& Rapoport, A. (2009). Excessive expenditure in two-stage contests: theory and experimental evidence. In I. N. Hangen \& S. Nilsen (Eds.), Game theory: strategies, equilibria, and theorems (pp. 241-266). Hauppauge: Nova Science Publishers.

Amegashie, J. A. (1999). The design of rent-seeking competitions: committees, preliminary and final contests. Public Choice, 99, 63-76.

Amegashie, J. A., Cadsby, C. B., \& Song, Y. (2007). Competitive burnout: theory and experimental evidence. Games and Economic Behavior, 59, 213-239.

Anderson, L. R., \& Stafford, S. L. (2003). An experimental analysis of rent seeking under varying competitive conditions. Public Choice, 115, 199-216.

Blavatskyy, P. (2004). Why qualifications at the Olympics? Institute for Empirical Research in Economics, University of Zurich, Unpublished manuscript.

Che, Y.-K., \& Gale, I. (1997). Rent-seeking when rent seekers are budget constrained. Public Choice, 92, $109-126$.

Che, Y.-K., \& Gale, I. (1998). Standard auctions with financially constrained bidders. Review of Economic Studies, 65, 1-21.

Che, Y.-K., \& Gale, I. (2003). Optimal design of research contests. American Economic Review, 93, 646-671.

Davis, D., \& Reilly, R. (1998). Do many cooks always spoil the stew? An experimental analysis of rent seeking and the role of a strategic buyer. Public Choice, 95, 89-115. 
Fey, M. (2008). Rent-seeking contests with incomplete information. Public Choice, 135, 225-236.

Gradstein, M. (1998). Optimal contest design: volume and timing of rent seeking in contests. European Journal of Political Economy, 14, 575-585.

Gradstein, M., \& Konrad, K. A. (1999). Orchestrating rent seeking contests. Economic Journal, 109, 536545.

Harbaugh, R., \& Klumpp, T. (2005). Early round upsets and championship blowouts. Economic Inquiry, 43, 316-329.

Harstad, R. M. (1995). Privately informed seekers of an uncertain rent. Public Choice, 83, 81-93.

Hillman, A., \& Riley, J. G. (1989). Politically contestable rents and transfers. Economics and Politics, 1, $17-40$.

Konrad, K. A. (2009). Strategy and dynamics is contests. Oxford: Oxford University Press.

Millner, E. L., \& Pratt, M. D. (1989). An experimental investigation of efficient rent seeking society. Public Choice, 62, 291-303.

Millner, E. L., \& Pratt, M. D. (1991). Risk aversion and rent seeking: an extension and some experimental evidence. Public Choice, 69, 91-92.

Moldovanu, B., \& Sela, A. (2001). The optimal allocation of prizes in contests. American Economic Review, $91,542-558$.

Moldovanu, B., \& Sela, A. (2006). Contest architecture. Journal of Economic Theory, 126, 70-96.

Önçüler, A., \& Croson, R. (2004). Rent seeking for a risky rent: a model and experimental investigation. Journal of Theoretical Politics, 17, 403-429.

Parco, J. A., Rapoport, A., \& Amaldoss, W. (2005). Two-stage contests with budget constraints: an experimental study. Journal of Mathematical Psychology, 49, 320-338.

Potters, J. C., de Vries, C., \& van Linden, F. (1998). An experimental examination of rational rent seeking. European Journal of Political Economy, 14, 783-800.

Schmitt, D., Shupp, R., \& Walker, J. M. (2003). Resource allocation contests: experimental evidence. Federal Trade Commission, Bureau of Economics, Unpublished manuscript.

Schmitt, P., Shupp, R., Swope, K., \& Cardigan, J. (2004). Multi-period rent-seeking contests with carryover: theory and experimental evidence. Economics of Government, 5, 187-211.

Shogren, J. F., \& Baik, K. H. (1991). Reexamining effective rent seeking in laboratory markets. Public Choice, 69, 69-97.

Stein, W. E. (2002). Asymmetric rent-seeking with more than two contestants. Public Choice, 113, 325-336.

Stein, W. E., \& Rapoport, A. (2004). Asymmetric two-stage group rent-seeking: comparison of two contest structures. Public Choice, 118, 151-167.

Stein, W. E., \& Rapoport, A. (2005). Symmetric multi-stage contests with budget constraints. Public Choice, 124, 309-328.

Vogt, C., Weimann, J., \& Yang, C.-L. (2002). Efficient rent-seeking in experiment. Public Choice, 110, 6778.

Wärneyrd, K. (2003). Information in conflicts. Journal of Economic Theory, 110, 121-136.

Weimann, J., Yang, C.-L., \& Vogt, C. (2000). An experiment on sequential rent seeking. Journal of Economic Behavior and Organization, 41, 405-426. 